\title{
Optimization Design for Ocean Wave Energy Convertor
}

\author{
Liu $\mathrm{Na}^{1, *}$, Tan Yimin ${ }^{2}$, MO Weiqiang ${ }^{1}$, HAN Huanqing ${ }^{1}$, LI Lin ${ }^{1}$ \\ ${ }^{1}$ Beijing Institute of Technology, Zhuhai, School of Industrial Automation, 519088 Guangdong, China \\ ${ }^{2}$ Sea Electric Energy Inc. Mississauga, L5N1L2 Mississauga, Canada
}

\begin{abstract}
Establishing a theoretical model for Ocean Wave Energy Convertor in advance is a necessary step during studying the energy harvesting of ocean wave which can save the engineering cost and improve research efficiency. Since low energy conversion efficiency existed in wave energy convertor when capturing ocean wave energy, the mechanism of slotless Halbach linear generator which can optimize the magnetic field distribution of the generator is adopted as the secondary energy conversion devices to solve the problem. The magnetic vector potential theory is introduced to analysis the topology of Halbach linear generator, then expressions of the generator's performance have been deduced. Hence, the analysis model of the Halbach linear generator has been settled. To obtain the global optimal solution, the simulated annealing algorithm has been used to slove that derived model. Then a series of linear generator's design parameters are fixed, which include dimensions of permanent magnets and winding coils. The error of linear generator's peak power between analytical solution results and simulation results is about $3.6 \%$. The experiment result demonstrates that maximum output power of optimized Halbach linear generator reaches 570w.
\end{abstract}

\section{Introduction}

With the development of the world economy and the grown of population, and the demand for energy is increasing. Countries have successively carried out research on new green energy. Ocean wave energy have received a lot of attention because of their characteristics of high energy density, wide distribution and inexhaustible[1]. The UK has built the Orkney Wave Power Test Site and manufactured the wave power generation equipment with power of $2 \mathrm{MW}$. A Scottish company has designed a wave energy convertor called Pelamis, and whose electricity capacity is nearly to $1 \mathrm{MW}$. A Swedish company has designed a type of onebody linear generator with vertical oscillating buoy, and whose output power can achieve 20kW. An American company has invented a two-body powerbuoy device generating electricity through the relative motion of two oscillating floats[2-4]. China's study on ocean wave energy harvesting mainly concentrates on the type of fixed and floating wave energy convertor[5]. Wanshan island power station in Zhuhai with installed electricity of $3 \mathrm{~kW}$ has been constructed, then the $20 \mathrm{~kW}$ shoremounted wave energy station has been built. The $8 \mathrm{~kW}$ pendulum type wave power station, the $100 \mathrm{~kW}$ shoremounted oscillating water column power station and the $30 \mathrm{~kW}$ pendulum type wave power station has been built one after another. For the time being, the research direction of ocean wave energy convertor mainly focuses on large-scale ocean wave convertor, while the research on small-scale ocean energy harvesting are less relatively[9]. In this paper, a comprehensive theoretical model of slotless Halbach linear generator is proposed to analyze the conversion of process of wave energy to electric energy, which can be used in researching smallscale ocean wave energy device and providing energy source for establishment of ocean buoy monitoring system.

During the process of designing wave energy convertor, some critical parameters need to be optimized. The study[10] found that several parameters have impact on performance of the linear generator, which are the length and thickness of the permanent magnet (PM), the central shaft radius, the width of air gap, the wire gauge of winding coil and the wave speed. The optimization of several parameters is a combinatorial optimization problem. There are some global optimization algorithms used to solve the problem, such as genetic algorithm, simulated annealing algorithm(SAA) and ant colony optimization algorithm[11]. In references[12-13], the genetic algorithm was adopted to solve the optimization design problem of permanent magnet linear motor, after that the performance of the motor was improved. Reference[14] applied ant colony algorithm to solve the optimal design of multi-megawatt wind turbines with Halbach Permanent magnet synchronous generator. Markovic adopted a software named ProDesign for optimal design to improve the power density, but the software only returned one output parameter[15]. Kirkpatrick et al.[16] introduced the idea of annealing into the field of combinatorial optimization and compared the combinatorial optimization problems with thermodynamic equilibrium issues in statistical mechanics.Consequently, an approximate global optimal

\footnotetext{
* Corresponding author: $43736176 @$ qq. com
} 
solution was obtained in the process of simulated annealing. The simulated annealing algorithm is able to achieve the balance between accuracy and efficiency. The algorithm can probabilistically jump over the trap of local optimal solution and eventually get an approximately global optimizaiton. Therefore, the SAA will be adopted to optimize the critical parameters of Halbach linear generator.

\section{Introduction of ocean wave energy convertor}

Harvesting ocean wave energy has various convertors with different configurations. There are oscillating water column, hydraulic transmission and direct-driven linear generator[17], as shown in Fig.1. In the third type, the convertor has a construction of two-body oscillating buoy and uses the Halbach linear generator as the secondary energy convertor. The linear generator produces electricity through relative motion between different components. The energy conversion process of directly driven can effectively decrease intermediate link mechanism and energy loss.

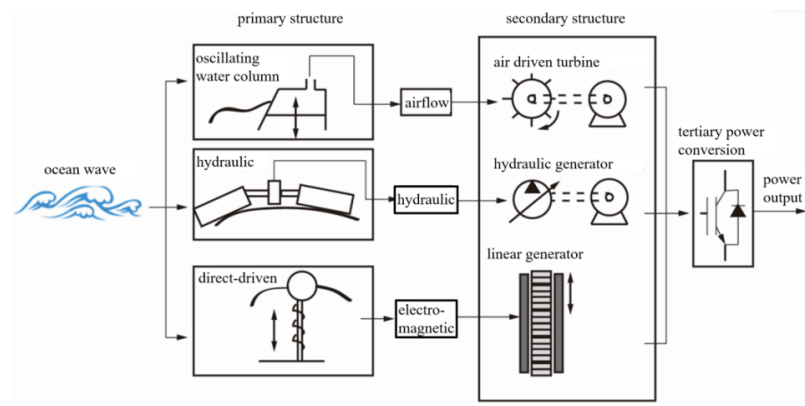

Fig. 1. Schematic of wave energy convertor

\section{Model of the Halbach linear generator}

\subsection{Topology}

To save engineering cost and increase efficiency of the research work, a theoretical model will be established before developing the specific equipment. Reference[18] indicates that when the input of wave force is $4 \mathrm{kN} \cdot \mathrm{m}^{2}$, and the output of the generator is $3 \mathrm{~kW}$. The reason is that conventional linear generators adopt slotted armature to decrease the air reluctance on purpose of improving the efficiency in electricity generation. While, the periodically changed cogging force (a type of magnetic resistance) introduced by slotted armature will greatly hinder the relative motion between generator's rotor and stator, and cogging force will lead to instability of the system. This design has adopted slotless armature to weaken detent force effectively and improve the stability of generator. In order to further improve efficiency of energy conversion, the permanent of wave energy convertor will use Halbach permanent magnet arrays.

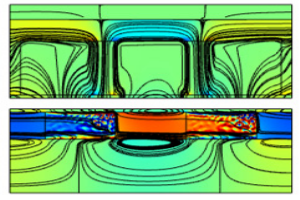

(a)

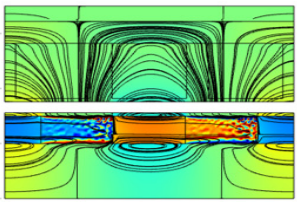

(c)

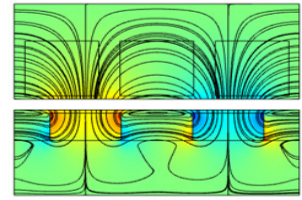

(b)

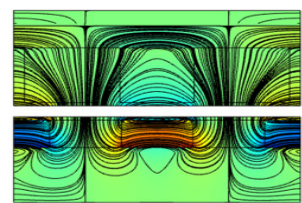

(d)
Fig. 2. Finite element calculation results of four types of magnetic field distributions

Fig.2 illustrates finite element calculation results of four types of magnetic field distribution. The simulation results respectively are slotted axial magnetization, slotless Halbach axial magnetization with coreless, slotted axial magnetization with core and slotless Halbach axial magnetization with core, as shown in Fig.2(a), (b), (c), (d). Hence, the design of slotless Halbach permanent magnet (PM) arrays can optimize the magnetic field distribution. The topology of Halbach linear generator is shown in Fig.3, where (a) is the 3D model of the linear generator and (b) is the generator's axial section.

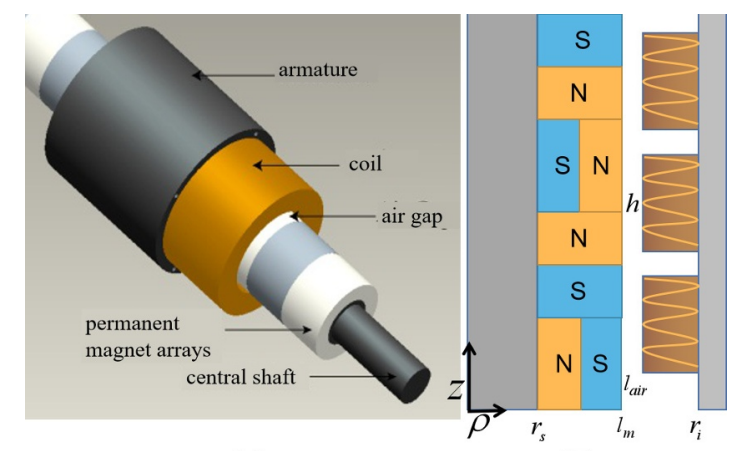

(a)

(b)

Fig. 3. The topology of Halbach linear generator

\subsection{The comprehensive analysis model}

According to Faraday's law of electromagnetic induction, the inductive electromotive force generated by the system can be written as:

$$
\mathrm{e}=-\frac{d \Psi}{d t}=-N \frac{d \Phi}{d t}
$$

where $\Psi=N \Phi$. The induced electromotive force of winding coil is proportional to it's turns and the change rate of magnetic flux[19]. In the process of establishing magnetic field distribution model, The three regions are air gap region - region 1, Halbach permanent magnet array region - region 2 and central shaft of armature region 3. The magnetic field strength of magnet and free space domain can be expressed as

$$
B=\left\{\begin{array}{l}
\mu_{0} H \\
\mu H+\mu_{0} M
\end{array}\right.
$$


where $\mu=\mu_{0} \mu_{\mathrm{r}}$, and $\mu_{0}$ represents permeability in vacuum, $\mu_{\mathrm{r}}$ represents permeability in magnetic. $\mathbf{H}$ and $B_{M}$ are respectively magnetic intensity and strength of the magnetized field, both of which are vector. The magnetic field is a curl field without divergence and magnetic induction lines are always closed, hence the divergence of magnetic induction intensity is always zero, i.e. $\nabla \cdot B=0$. According to the vector analysis theory, introducing vector $v$ to equation(2), then equation(3) can be written as:

$$
\left\{\begin{array}{l}
\nabla^{2} \cdot v_{1,3}=0 \\
\nabla^{2} \cdot v_{2}=-\mu_{0} \cdot \nabla \cdot B_{M}
\end{array}\right.
$$

The magnetized magnetic density can be solved by Fourier transform,

$$
\left\{\begin{array}{l}
B_{M \rho}=\sum_{j=-\infty}^{\infty}(-1)^{\frac{j-1}{2}} \frac{4 B_{0}}{\mu_{0}} \frac{\sin \frac{j \pi}{4} \sin \frac{j \pi}{2}}{j \pi} \cos \left(n_{j} z\right) \\
B_{M z}=\sum_{j=-\infty}^{\infty}(-1)^{\frac{j+1}{2}} \frac{4 B_{0}}{\mu_{0}} \frac{\sin \frac{j \pi}{4}}{j \pi} \cos \left(n_{j} z\right)
\end{array}\right.
$$

where $B_{M \rho}$ and $B_{M z}$ are radial magnetic density and axial magnetic density of Halbach PM arrays in cylindrical coordinates system, respectively. And $n_{j}=\frac{2 j \pi}{L_{m}}$, where $n_{j}$ is the jth spatial angular frequency of the harmonic component.

In cylindrical coordinates, the vector $v$ has only one component, i.e. $v_{\theta}$. Hence, equations of corresponding regions can be obtained as follow:

$$
\left\{\begin{array}{l}
\frac{\partial^{2} v_{1 \theta}}{\partial z^{2}}+\frac{\partial^{2} v_{1 \theta}}{\partial \rho^{2}}+\frac{1}{\rho} \frac{\partial v_{1 \theta}}{\partial \rho}-\frac{\partial v_{1 \theta}}{\partial \rho^{2}}=0 \\
\frac{\partial^{2} v_{2 \theta}}{\partial z^{2}}+\frac{\partial^{2} v_{2 \theta}}{\partial \rho^{2}}+\frac{1}{\rho} \frac{\partial v_{2 \theta}}{\partial \rho}-\frac{\partial v_{2 \theta}}{\partial \rho^{2}}=-\mu_{0} \frac{\partial B_{M \rho}}{\partial z} \\
\frac{\partial^{2} v_{3 \theta}}{\partial z^{2}}+\frac{\partial^{2} v_{3 \theta}}{\partial \rho^{2}}+\frac{1}{\rho} \frac{\partial v_{3 \theta}}{\partial \rho}-\frac{\partial v_{3 \theta}}{\partial \rho^{2}}=0
\end{array}\right.
$$

the continuity of boundaries between regions 1 and 2, 2 and 3 should satisfy the following conditions:

$$
\left\{\begin{array}{l}
\frac{B_{1 z}}{\mu_{0}}=\frac{B_{2 z}}{\mu_{0}}-H_{1 z}, \quad B_{1 \rho}=B_{2 \rho} \\
\frac{B_{3 z}}{\mu_{0}}=\frac{B_{2 z}}{\mu_{0}}-H_{3 z}, \quad B_{3 \rho}=B_{2 \rho}
\end{array}\right.
$$

Consequently, the magnetic flux density of regions 1 and 3 are 0 . By solving boundary condition equations and regional equations, expressions of magnetic field intensity of three regions can be written as follows:

$$
\left\{\begin{aligned}
B_{1 \rho}= & -n_{k}\left(C_{1 k} I_{1}\left(n_{k} \rho\right)\right) \cos \left(n_{k} z\right) \\
B_{1 z}= & n_{k}\left(C_{1 k} I_{0}\left(n_{k} \rho\right)\right) \sin \left(n_{k} z\right) \\
B_{2 \rho}= & -\left(n_{k} C_{3 k}+F_{A K}\left(n_{k} \rho\right)\right) I_{1}\left(n_{k} \rho\right) \cos \left(n_{k} \rho\right) \\
& -\left(n_{k} C_{4 k}-F_{B k}\left(n_{k} \rho\right)\right) K_{1}\left(n_{k} \rho\right) \cos \left(n_{k} \rho\right) \\
B_{2 z}= & \left(n_{k} C_{3 k}+F_{A K}\left(n_{k} \rho\right)\right) I_{0}\left(n_{k} \rho\right) \sin \left(n_{k} \rho\right) \\
& -\left(n_{k} C_{4 k}-F_{B k}\left(n_{k} \rho\right)\right) K_{0}\left(n_{k} \rho\right) \sin \left(n_{k} \rho\right) \\
B_{3 \rho}= & -n_{k}\left(C_{5 k} I_{1}\left(n_{k} \rho\right)\right)+C_{6 k} K_{1}\left(n_{k} \rho\right) \cos \left(n_{k} z\right) \\
B_{3 z}= & n_{k}\left(C_{5 k} I_{0}\left(n_{k} \rho\right)\right)-C_{6 k} K_{0}\left(n_{k} \rho\right) \sin \left(n_{k} z\right)
\end{aligned}\right.
$$

where $\mathrm{I}$ and $\mathrm{K}$ are the modified bessel function of the first and the second function, respectively. Thus, the flux and reaction force of one single coil can be written as:

$$
\begin{gathered}
\Psi=-\frac{N}{h\left(r_{i}-l_{m}-g\right)} \int_{z}^{z+h} \int_{l_{m}+l_{\text {air }}}^{r_{i}} 2 \pi \rho \sigma_{1}(\rho, z) d_{\rho} d_{z} \\
F=-\int_{z}^{z+h} \int_{l_{m}+l_{\text {air }}}^{r_{i}} 2 \pi \rho B_{2 \rho}(\rho, z) d_{\rho} d_{z}
\end{gathered}
$$

where $l_{\text {air }}$ is the width of air gap, $h$ is the height of each single winding coil. In equation (9), $F$ is regarded as a parameter related to the damping, then generator's power take-off(PTO) can be obtained. Then the equivalent damping coeffiecient of PTO system will be defined as equation (10), where $v$ is the velocity of ocean wave.

$$
C_{\text {PTO }}=\frac{d F}{d v}
$$

Assuming that the effective value of induction electromotive force (EMF) is E, the output power and output voltage of the Halbach linear generator can be expressed as follows:

$$
\left\{\begin{array}{l}
V=\frac{E}{R_{o}+R_{i}} R_{o} \\
P=\left(\frac{E}{R_{o}+R_{i}}\right)^{2} R_{o}
\end{array}\right.
$$

where $R_{i}$ is the coil's internal impedance, and $R_{o}$ is the generator's external load. When the condition $R_{o}=R_{i}$ is satisfied, the maximum power can be achieved.

\section{Study on the model of Halbach linear generator}

\subsection{Effect of winding gauge on Halbach linear generator's output power}

It's generally considered that selection of winding gauge will impact on output power of the generator. Under the same winding topology, the coil's turns is inversely proportional to square of wire diameter, i.e. $N=\frac{S}{d^{2}}$. And the EMF is determined by the turns while keeping other parameters be constant. The total resistance of winding coils is given by

$$
R_{i}=R_{\rho} \frac{L}{A}=\frac{8 \pi r_{\text {coil }} R_{\rho} N}{\pi d^{2}}
$$

Where $r_{c o i l}$ is the coil's radius, $R_{\rho}$ is the relative resistance, and $d$ is wire diameter. From aforementioned equations (1) and (8), the maximum output power of coils can be expressed as follows:

$$
P(\mathrm{t})=\frac{E^{2}}{4 R_{i}}=\frac{\left(\frac{d \Phi}{d t}\right)^{2} d^{2} N}{32 r_{\text {coil }} R_{\rho}}=\frac{\left(\frac{d \Phi}{d t}\right)^{2} S}{32 r_{\text {coil }} R_{\rho}}
$$

From equation (13), we can deduced that the output power is independent of the AWG selection. But it's undeniable that the diameter of the winding coil has a significant influence on the current which the linear generator can withstand. 


\subsection{Effect of permanent magent's thickness on Halbach linear generator's output power}

According to the theoretical model established in the paper's second part, the PM thickness of Halbach linear generator $l_{m}$ is a parameter affecting directly the value of flux $\Psi$ and reaction force $F$. In order to find the relationship between $l_{m}$ and output power of ther generator, the following parameters should be specified which are length of four-segments Halbach PM $l_{t}$, central shaft raidus $r_{s}$, air gap width $l_{\text {air }}$ and coil's AWG. Fig.4 shows that the peak power of the linear generator increases monotonously as PM thickness thicker. The reason is that magnetic density distribution in the air gap is enhanced with the thickness of permanent magnet becoming greater. Correspondingly, the output power of the linear generator has also increased.

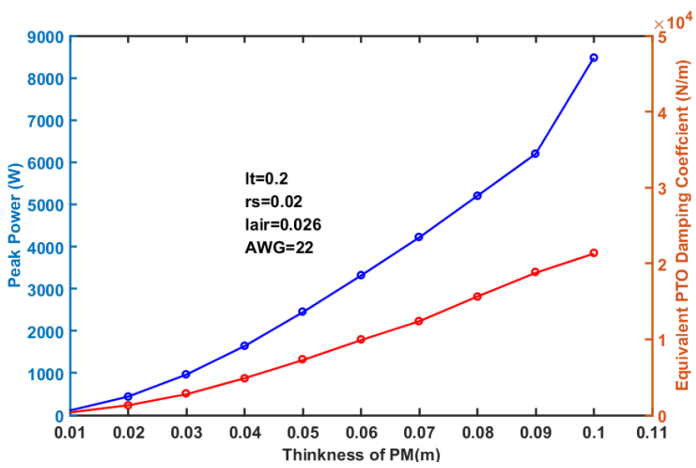

Fig. 4. The relationship between Halbach linear generator's peak power and the thickness of permanent magnet.

\subsection{Effect of the central shaft's radius on Halbach linear generator's output power}

Fig.5 indicates that the peak power of the Halbach linear generator is also increasing monotonously as $r_{s}$ increasing with the value of $l_{t}, l_{m}, l_{\text {air }}$ and AWG are specified as the constants.

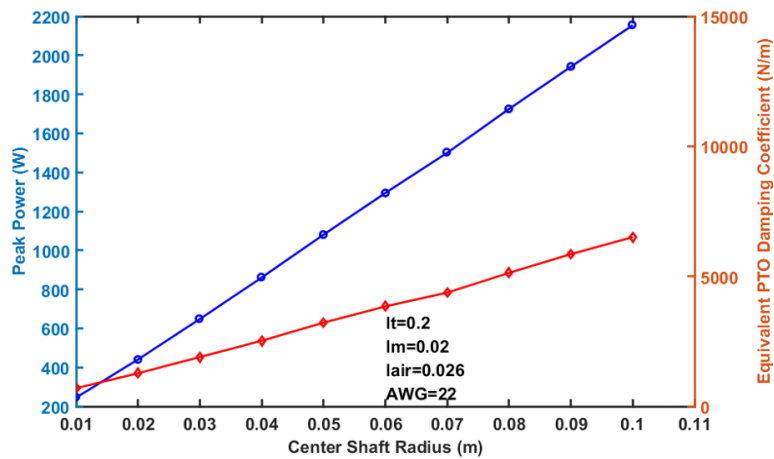

Fig. 5. The relationship between Halbach linear generator's peak power and the radius of central shaft.

Besides, it can be observed from Fig.4 and Fig. 5 that equivalent damping coefficient of the linear generator is increasing monotonously with increasement of $l_{m}$ and $r_{s}$. The reason is that after the central shaft radius and the PM thickness of the generator are getting larger in size, the magnetic distribution will be enhanced and the magnetomotive force becomes larger. In addition, the equivalent PTO damping coefficient will also increase. Additionally, the structure of the generator will become cumbersome as its size becoming larger, which also will raise the processing costs and complicate assembly. Actual requiremnts and occasion demands ought to be considered.

\subsection{Magnetic density distribution of Halbach linear generator theoretical model}

To testify the theoretical model of Halbach linear generator, simulated calculation and analytical calculation were performed respectively with COMSOL and MATLAB, as shown in Figures 6-8. The maximum magnetic flux density of PM arrays on the $z$ axial direction is $0.75 \mathrm{~T}$, and the maximum magnetic flux of $\mathrm{PM}$ arrays on the $\rho$ axial direction reaches 1.4T. This topology can greatly suppresses the magnetic flux density distribution on the $z$ axial.

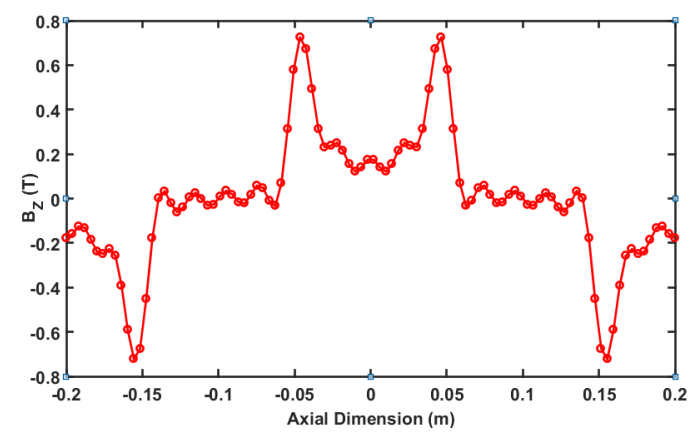

Fig. 6. Magnetic density distribution of PM arrays on $\mathrm{z}$ axis

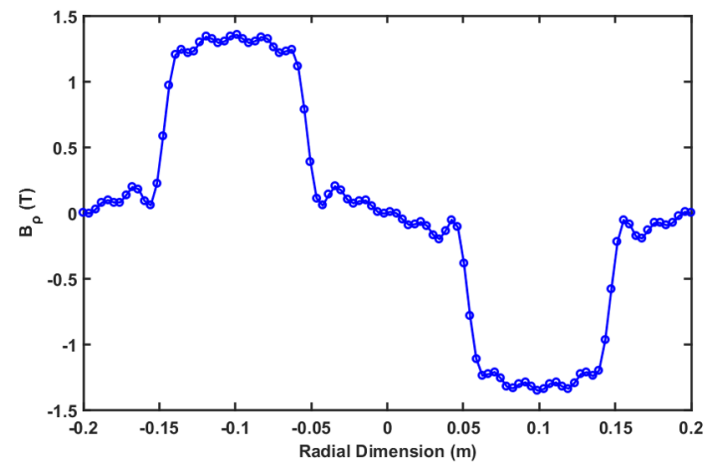

Fig. 7. Magnetic density distribution of PM arrays on $\rho$ axis

The magnetic field distribution of Halbach linear generator is shown in Figure 8. It is found that the finite element simulation result is basically match with the analytical calculation result.

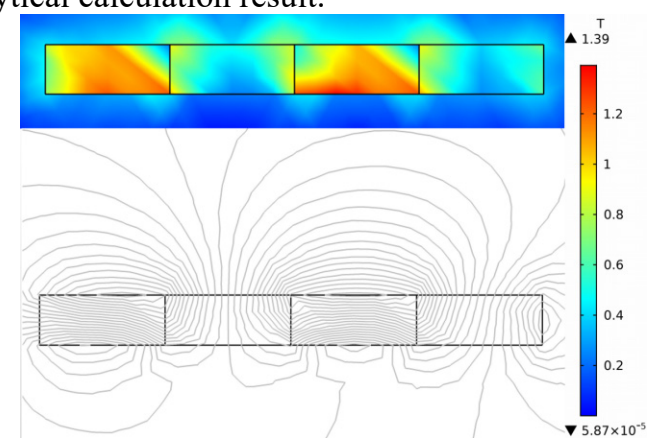

Fig. 8. Magnetic density distribution of PM arrays for Halbach linear generator 


\section{Application of simulated annealing algorithm}

\subsection{Flowchart of simulated annealing algorithm}

The SAA adopts Metropolis acceptance criterion and a cooling schedule consisted of four parameters to control the process of the algorithm. The process of iterative was started from a certain initial value, and then generate a new solution. Subsequently, accept or reject the new value with a specified probability. Parameters of cooling schedule are initial value $t_{i}$, end value $t_{e}$, length of the Markov Chain $L_{k}$ and decay function $\alpha$ [20].

According to the experiment conclusion by Johnson et al. [21], the value of $t_{i}$ was defined as $10^{4}$. The stopping criterion constructed by Nahar et al. indicates that high quality eventual solution can be obtained only if control parameter is insignificant compared with its initial value[22]. Therefore, the eventual value of control parameter is defined as 0.01 . And the length of Markov Chain is fixed as 200. As for control parameter's decay function, a small attenuation will make the algorithm process access more neighbourhoods and return higher quality eventual solution. $t_{i+1}=\alpha \cdot t_{i}$ is a common decay function of the control parameter, which was primarily proposed by Kirkpatrick et al.[16]. The $\alpha$ was settled to 0.95 in that paper. To keep quality of eventual solution without affecting CPU time rationality, the value of $\alpha$ is defined as 0.95. The flowchart of SAA is shown in Fig.9.

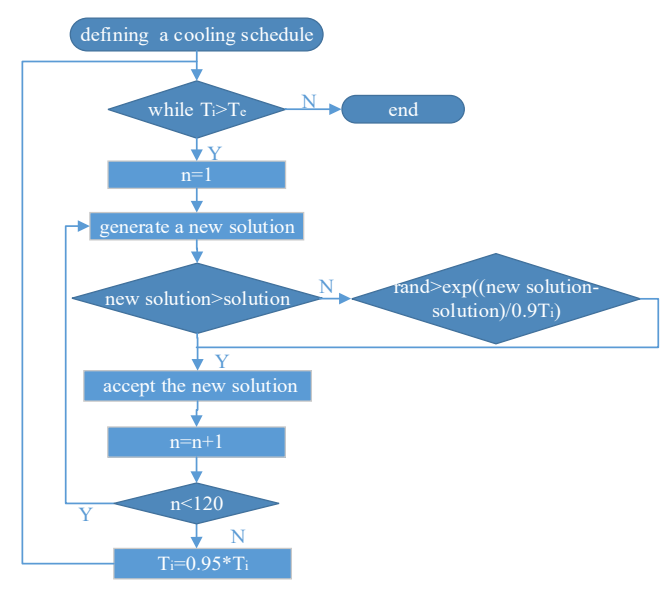

Fig. 9. The flow chart of Simulated Annealing Algorithm

\subsection{Verification for Halbach linear generator model}

Solving the theoretical model, the maximum power of Halbach linear generator is 504W. The result can be gained by observing Fig. 10.

From Fig.11, the maximum power of the Halbach linear generator is $527.24 \mathrm{~W}$. Generator's parameters are settled, which are the width of air gap is $6 \mathrm{~mm}$, the length of four-segments Halbach permanent magnetic (PM) arrays is $392 \mathrm{~mm}$, the radius of central shaft is $20 \mathrm{~mm}$, thickness of the PM is $48 \mathrm{~mm}$, the height of one single coil is $40 \mathrm{~mm}$.

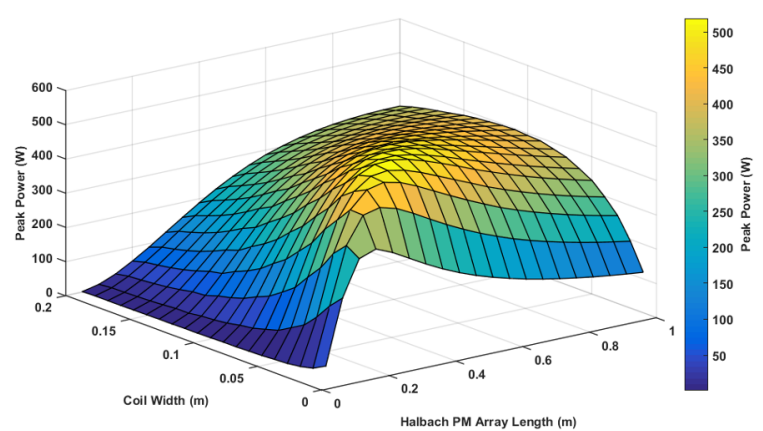

Fig. 10. The peak power of a single phase coils

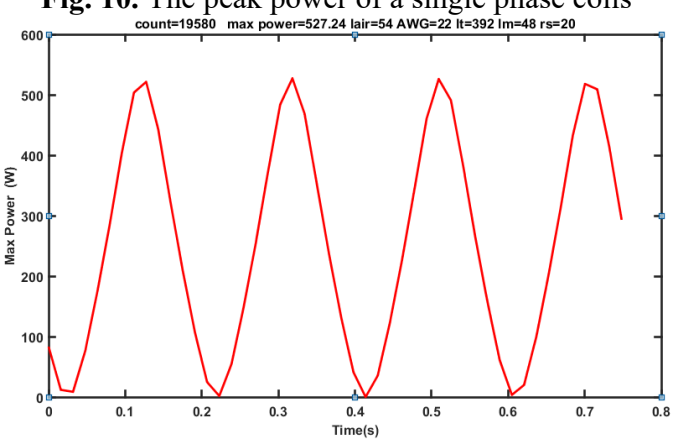

Fig. 11. Result of simulated annealing algorithm

The error between the SAA's result and the analytical solution's is within $3.6 \%$. They are basically matched. Corresponding parameters of the Halbach linear generator are shown in Table1.

Table 1. Design parameters of the Halbach linear generator

\begin{tabular}{c|c}
\hline Parameters & Value \\
\hline $\begin{array}{c}\text { width of the air gap } \\
\text { length of four-segments Halbach PM } \\
\text { arrays }\end{array}$ & $6 \mathrm{~mm}$ \\
\hline radius of central shaft & $20 \mathrm{~mm}$ \\
\hline thickness of the PM & $48 \mathrm{~mm}$ \\
\hline height of one single coil & $67 \mathrm{~mm}$ \\
\hline
\end{tabular}

\section{Conclusion}

The Halbach linear generator's prototype is shown in Figure 12. The inner diameter of PM arrays is $40 \mathrm{~mm}$, the outer diameter is $80 \mathrm{~mm}$, and the height is $50 \mathrm{~mm}$. All magnets are assembled on a ring aluminum central shaft. Three-phase coils are wrapped by an armature made of silicon steel. The inner diameter of each single phase coils is $92 \mathrm{~mm}$, the outer diameter is $132 \mathrm{~mm}$, and the height is $67 \mathrm{~mm}$. The linear actuator controlled by Simulink simulates the force of the waves, and it provides speeds up to $1.2 \mathrm{~m} / \mathrm{s}$. The position sensor is used to measure relative movement and provide feedback signals to the linear actuator. A force sensor is used in the prototype to measure the reaction force. When the impedance of the load and the coils matches, the average output power of the prototype achieves $142 \mathrm{~W}$ at a relative speed of $0.53 \mathrm{~m} / \mathrm{s}$. When the speed is $1 \mathrm{~m} / \mathrm{s}$, the total maximum output power of the system is expected to achieve $570 \mathrm{~W}$. 


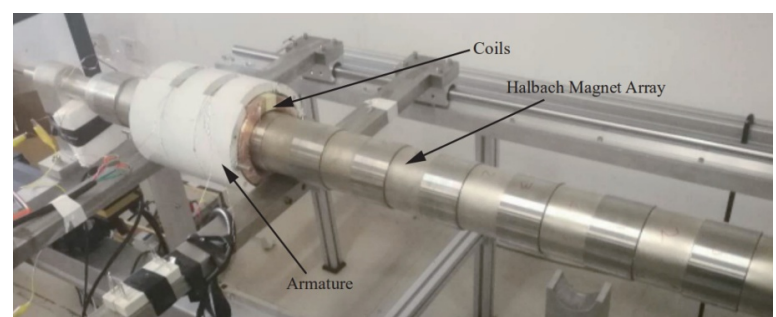

Fig. 12. Prototype for Halbach linear generator

This paper presents the topology of Halbach linear generator and analysis several selected parameters which have impact on the magnetic density distribution. Then using SAA to gain a group of design parameters and the maximum output power of the small-scale ocean wave convertor. Additionally, the flux density in the air gap is up to $1.4 \mathrm{~T}$ in this design. Excessive magnetic field density will make generator lead to supersaturation. That is worth noting in the experiment.

\section{References}

1. You Yage, Li Wei, Liu Weimin, et al. Development Status and Perspective of Marine Energy Conversion Systems[J]. Automation of Electric Power Systems,2010(14): 1-12.

2. Elie Al Shami, Ran Zhang, Xu Wang. Point Absorber Wave Energy Harvesters: A Review of Recent Developments[J]. Energies,2018,12(1).

3. Marcus Lehmann, Farid Karimpour, Clifford A. Goudey, Paul T. Jacobson, Mohammad-Reza Alam. Ocean wave energy in the United States: Current status and future perspectives[J]. Renewable and Sustainable Energy Reviews,2017,74.

4. Engström J, Kurupath V, Isberg J, et al. A resonant two body system for a point absorbing wave energy convertor with direct-driven linear generator[J]. Journal of applied physics, 2011, 110(12): 124904.

5. Henderson R. Design, simulation, and testing of a novel hydraulic power take-off system for the Pelamis wave energy convertor. Renewable Energy 2006;31:271-83.

6. Chen Renwen, Liu Chuan, Zhang Yuxiang. Research Status and Perspective of Direct Ocean Wave Energy Harvesters[J]. Journal of Data Acquisition\& Processing, 2019,34(02):195-204.

7. Wang Xin, Li Daming, Li Xuelin, et al. Analysis on the Hydrodynamic Impact Among the Berths in the Dawanshan Wave Energy Test Site[J]. Journal of Ocean Technology, 2015, 34(04):69-73.

8. E Shiju, Jin Jianhua, Cao Jianbo, et al. Development of Permanent Magnet Linear Synchronous Motors Structure and Research [J]. Journal of Mechanical \& Electrical Engineering, 2016, 33(12): 1421-1427.

9. Mao Yaofei, Zhu Keqiang, Zhou Chen, et al.Tentative Identification for Design Parameters of Oscillating Floater Buoy Wave Energy convertor [J]. Ship Engineering, 2017,39(03):65-69+88.

10. Zhang Chunlei, Zhang Hui, Ye Peiqing, et al. Research on Sensorless Algorithm of Two-Phase
Tubular Permanent Magnet Synchronous Linear Motor.[J]. Transactions of China Electrotechnical Society, 2019,34(23):4901-4908.

11. Lu Qinfen, Shen Yiming, Ye Yongyue. Development of Permanent Magnet Linear Synchronous Motors Structure and Research[J]. Proceedings of The Chinese Society for Electrical Engineering,2019,39(09): 2575-2588.

12. Xu Xiaozhuo, Sun Zhen, Wang Xudong et al. Characteristic of a Novel Permanent Magnet Linear Synchronous Motor with Halbach Array Consequent-Pole[J]. Transactions of China Electrotechnical Society, 2019, 34(09): 1825-1833.

13. EIGebaly, A. E., \& El-Nemr, M. K. Optimal Design of Slotless PM Halbach Array Linear Generator for Wave Energy convertors at Maximum Power Transfer Condition. In 2018 Twentieth International Middle East Power Systems Conference (MEPCON), 2018, (10):271-276).

14. Alshibani, S. Application of Particle Swarm Optimization in the Design of Halbach Permanent Magnet Synchronous Generators for Megawatt Level Wind Turbines. In 2018 7th International Conference on Renewable Energy Research and Applications (ICRERA), 2018, 10: 865-868.

15. Markovic, M., \& Perriard, Y. Optimization design of a segmented Halbach permanent-magnet motor using an analytical model. IEEE Transactions on Magnetics, 2009, 45(7), 2955-2960.

16. Kirkpatrick S, Gelatt C D, Vecchi M P, et al. Optimization by simulated annealing.[J]. Science, 1983, 220(4598): 671-680.

17. Tan $\mathrm{Y}, \mathrm{Lin} \mathrm{K}, \mathrm{Zu} \mathrm{J} \mathrm{W.} \mathrm{Analytical} \mathrm{modelling} \mathrm{of}$ Halbach linear generator incorporating pole shifting and piece-wise spring for ocean wave energy harvesting[J]. AIP Advances, 2018, 8(5): 056615.

18. Xiao Xi, Bai Nianzong, Kang Qing, et al. A Review of the Development of Wave Power System and the Research on Direct-Drive Wave Power System[J]. Electric Engineering magazine, 2014, 29(03):1-11.

19. Tan Yimin, Lin Kejian, and Zhang Zuguang. Comprehensive Modelling of A Slotless Halbach Linear Generator Based Wave Energy convertor, Proceedings of 44th Annual Conference, IEEE Industrial Electronics Society (IECON), Washington DC, 2018.

20. Kang Lishan, Xie Yun, You Shiyong, et al. Nonnumerical parallel algorithm[M]. Beijing: Science Press, 2003.

21. Johnson, D. S., Aragon, C. R., McGeoch, L. A., \& Schevon, C. Optimization by simulated annealing: An experimental evaluation; part I, graph partitioning. Operations research, 1989, 37(6):865892.

22. Nahar, S., Sahni, S. and Shragowitz. E. Experiments with Simulated Annealing, Proc.22nd Des. Automation Conf., Las Vegas, 1985(7), pp:748-752. 